\title{
Novedades en el Género Dahlia (Asteraceae: Coreopsideae) en Nueva Galicia, MéXico
}

\author{
Arturo Castro-Castro, Osvaldo Zuno-Delgadillo, Marco Antonio Carrasco-Ortiz, \\ Mollie Harker y Aarón RodríGueZ ${ }^{1}$ \\ Instituto de Botánica, Departamento de Botánica y Zoología, Centro Universitario de Ciencias Biológicas \\ y Agropecuarias, Universidad de Guadalajara, Zapopan, Jalisco, México \\ ${ }^{1}$ Autor para la correspondencia: rca08742@cucba.udg.mx
}

\begin{abstract}
Resumen: La tribu Coreopsideae (Asteraceae) concentra una alta diversidad y endemismo en el área conocida como Nueva Galicia, en el occidente de México. Dahlia es un género característico de Coreopsideae, y en Nueva Galicia se sabía que crecían D. barkerae, D. coccinea, D. pugana, D. scapigera, D. sherffii y D. tenuicaulis. En el presente trabajo, se registra la presencia de $D$. sorensenii y se propone a $D$. wixarika como especie nueva para la ciencia. La especie nueva es exclusiva del sur del estado de Durango y norte de Jalisco, presenta morfología afín a las secciones Dahlia y Entemophyllon, y tiene semejanzas morfológicas con D. dissecta, D. pugana y D. scapigera, con las cuales se compara. Además, se aportan datos nuevos relacionados con la distribución geográfica y macromorfología de $D$. pugana. Por último, se incluye una clave para la identificación de las especies presentes en Nueva Galicia.
\end{abstract}

Palabras clave: endemismo, Heliantheae, Sierra de los Huicholes, Sierra Madre Occidental.

Abstract: A considerable diversity and endemism of the tribe Coreopsideae (Asteraceae) is concentrated in western Mexico, a territory known as Nueva Galicia. Dahlia is a notable genus of Coreopsideae, and so far D. barkerae, D. coccinea, D. pugana, $D$. scapigera, $D$. sherffi, and D. tenuicaulis were registered for Nueva Galicia. In the present work, we report the presence of $D$. sorensenii and propose $D$. wixarika as a new species. The new taxon is exclusive to the south of the state of Durango and north of Jalisco. It has morphological affinities with sections Dahlia and Entemophyllon, and it is morphologically similar to D. dissecta, $D$. pugana, and D. scapigera, with which it is compared. In addition, we provide new data on geographical distribution and morphological variation of D. pugana. Finally, an identification key for the species of Dahlia in Nueva Galicia is provided.

Key words: endemism, Heliantheae, Sierra de los Huicholes, Sierra Madre Occidental.

$\mathbf{E}_{\mathrm{t}+\mathrm{m}}$ género Dahlia Cav. (Asteraceae: Coreopsideae) fue introducido a Europa a finales del siglo XVIII (Hansen y Hjerting, 1996, 2000). Ciento cuarenta años después, la lista de variedades de cultivo superaba 14,000 (Sorensen, 1970). En promedio, este número representa la designación de 100 cultivares por año desde que Dahlia fue llevada para su cultivo a España (Gatt et al., 1998). Ahora se reconocen entre 20,000 y 50,000 variedades de cultivo (Treviño de Castro et al., 2007), lo que ha posicionado a Dahlia como uno de los grupos de plantas ornamentales más conocidos, manejados y apreciados en el mundo.

Dahlia también ha generado interés desde el punto de vista taxonómico, biogeográfico y filogenético. Sorensen (1969) reconoció 27 especies y cuatro taxones infraespecífi- cos agrupados en cuatro secciones: Dahlia, Entemophyllon P.D. Sorensen, Epiphytum Sherff y Pseudodendron P.D. Sorensen. Sin embargo, hallazgos posteriores han ampliado el grupo a 37 especies (Sørensen, 1980, 1987; Saar y Sørensen, 2000, 2005; Saar, 2002; Saar et al., 2002, 2003b; Hansen y Sørensen, 2003; Castro-Castro et al., 2012). Por otro lado, con base en caracteres moleculares (Gatt et al., 2000; Saar et al., 2003a) y químicos (Giannasi, 1975), se ha encontrado que solo la sección Entemophyllon constituye un grupo natural, de modo que la monofilia del resto de las secciones sigue en discusión.

México representa el centro de diversificación de Dahlia (Sorensen, 1969; Castro-Castro et al., 2012; Carrasco-Ortiz, 2013). El mayor número de especies se concentra en el cen- 
tro y sur del país, en los estados de Hidalgo (12 spp.), Oaxaca (12), Guerrero (11), Querétaro (10), Michoacán (9), Estado de México (8) y Guanajuato (8) (Carrasco-Ortiz, 2013). Otra área con una diversidad considerable, es el territorio occidental de México denominado como Nueva Galicia, que comprende a los estados de Aguascalientes, Colima y Jalisco y porciones de los estados de Durango, Guanajuato, Michoacán, Nayarit y Zacatecas (McVaugh, 1961, 1972; Figura 1). Para esta área, McVaugh (1984), Castro-Castro et al. (2012) y Carrasco-Ortiz (2013) registraron la presencia de D. barkerae Knowles \& Westc., D. coccinea Cav., D. pugana Aaron Rodr. \& Art. Castro, D. scapigera (A. Dietr.) Knowles \& Westc., D. sherffii P.D. Sorensen y D. tenuicaulis P.D. Sorensen. No obstante, en el presente documento se registra la presencia de dos especies más; una de ellas se propone como especie nueva para la ciencia. También se aportan datos que amplían la distribución geográfica y la variación macromorfológica de $D$. pugana.

\section{Materiales y métodos}

Una primera tarea consistió en la revisión e identificación de ejemplares de Dahlia depositados en los herbarios CIMI, CIIDIR, CREG, GUADA, IBUG, IEB, MEXU y ZEA (acrónimos según Thiers, 2013). Con la información contenida en los ejemplares de herbario se elaboró una base de datos taxonómicos, geográficos, ecológicos y curatoriales. Además, se realizó un trabajo de campo durante los veranos de los años 2010 al 2013, que incluyó numerosas salidas de exploración y recolección de las especies de Dahlia presentes en Nueva Galicia. La información obtenida fue analizada con el Sistema de Información Geográfica ArcView Gis 3.3 (ESRI, 2002), y mapas de distribución geográfica fueron obtenidos para las especies de Dahlia circunscritas a Nueva Galicia.

\section{Resultados}

Nuevos registros. Dahlia tiene una distribución geográfica amplia en Nueva Galicia. Además de las seis especies citadas por McVaugh (1984), Castro-Castro et al. (2012) y Carrasco-Ortiz (2013), los siguientes taxones representan nuevos registros y elevan la cifra de especies a ocho.

Dahlia sorensenii H.V.Hansen \& Hjert., Nordic J. Bot. 16: 454. 1996.

Hierba perenne de 1.0-2.2 m alt.; raíces tuberosas, fasciculadas, fusiformes; tallo fistuloso, estriado, simple, pubescente solo en los nudos, hasta de $1.5 \mathrm{~cm}$ de diám., entrenudos de 5.5-29.0 cm long.; hojas opuestas, elíptico-ovadas a truladas en contorno general, simples, pinnadas o las inferiores a menudo bipinnadas y las superiores con frecuencia trifolioladas, 11.0-35.0 × 10.5-28.5 cm en hojas pinnadas, 7.0-17.0 $\times 4.0-11.0 \mathrm{~cm}$ en hojas simples; pecíolo sólido 2.0-12.0 $\times$

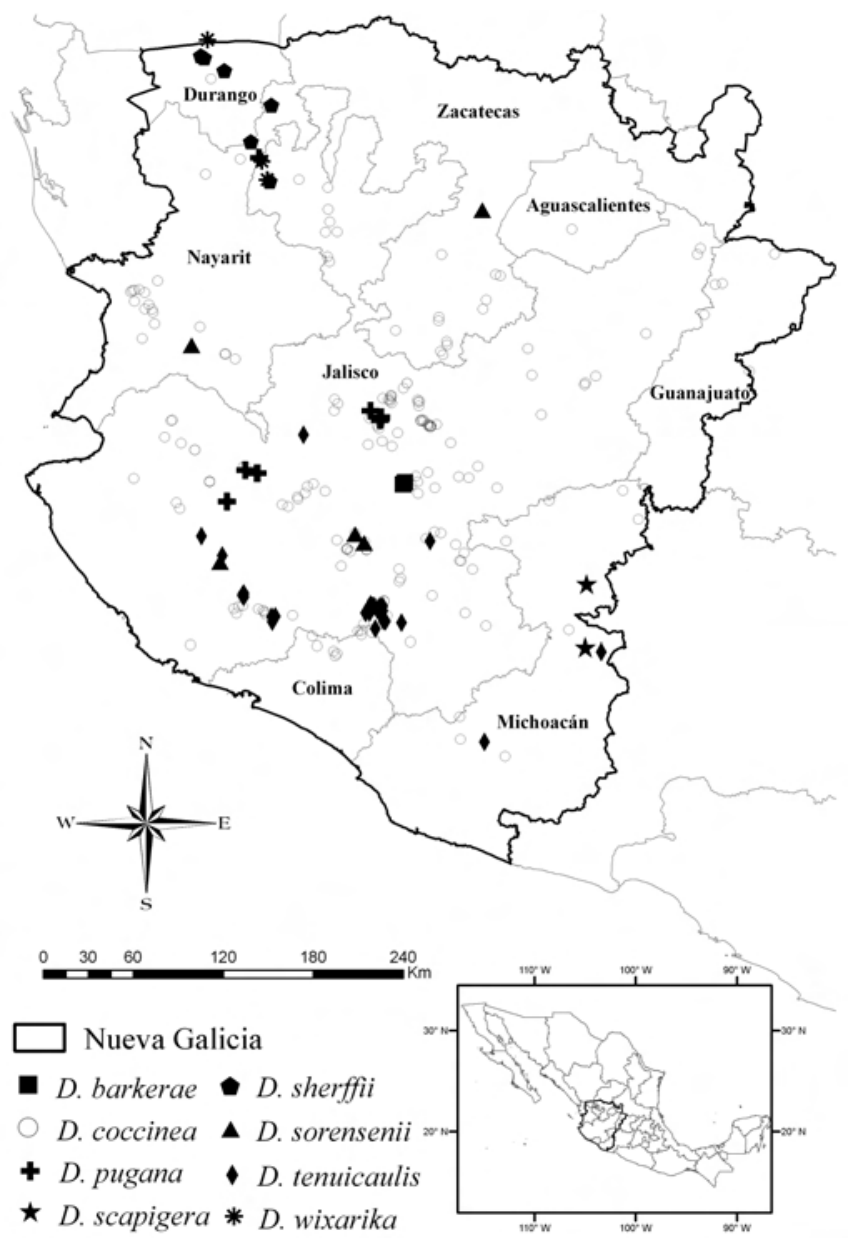

Figura 1. Distribución del género Dahlia en Nueva Galicia, México.

0.2-0.5 cm, prácticamente ausente en las hojas superiores, por lo general alado, sobre todo en la porción distal, con pubescencia similar a la del tallo, raquis alado; estipelas ausentes o presentes en el primer y segundo par de segmentos, 1.0-4.5 × 0.4-1.4 cm; folíolos 3-5(-9), sésiles y decurrentes en la base, elíptico-ovados, 3.5-17.0 × 1.5-11.0 cm, agudos o apiculados, atenuados o cuneados, serrados, de textura por lo general cartácea y rígida, escabrosos en ambas superficies, con pelos estrigosos, de color verde-oscuro en el haz, pálidos en el envés, con los pelos mayormente concentrados a lo largo de las nervaduras; foliólulos elípticos, 5.0-9.5 $\times$ 2.5-4.5 cm, atenuados a cuneados, agudos, serrados, ciliados; capítulos solitarios o 2-9 en los extremos de las ramas, pedúnculos 5.0-26.0 cm long., en su mayor parte glabros, pubescentes en su base; involucro campanulado, glabriúsculo; filarias exteriores 5(-7), oblongas, oblanceoladas u obovadas, $1.0-2.5 \times 0.3-0.5 \mathrm{~cm}$, verdes, reflejas o extendidas en la antesis, las interiores 8 , oblongas a elípticas, de 1.5-2.2 $\times 0.5-1.0 \mathrm{~cm}$, de color verde-amarillento en fresco y café con los márgenes, amarillos o hialinos al secar; páleas similares a las filarias interiores pero más pálidas; flores del 
margen 8(-9), liguladas, estériles, sus láminas elípticas a oblongas, ovadas u obovadas, de 2.0-6.0 × 1.0-2.0 cm, de color morado o violáceo, rara vez blanco, a veces más pálidas en la porción basal, glabras; flores del disco 90-145, tubulosas, de 10-12 mm long., de color amarillo, glabras, lóbulos de menos de $1.0 \mathrm{~mm}$ long., extendidos; anteras $c a$. $5.0 \mathrm{~mm}$ long., de color café; ramas del estilo con los ápices subulados, pubérulas por fuera; aquenios linear-oblanceolados, 11.0-13.0 mm long., negruzcos; vilano ausente; receptáculo plano a ligeramente convexo, 0.5-0.8 mm diám.

Distribución y ecología. Dahlia sorensenii tiene una distribución geográfica amplia en México. Crece sobre las vertientes norte y sur de la Faja Volcánica Transmexicana, desde el Estado de México y Morelos, hasta Jalisco y Nayarit. También se le encuentra en la vertiente oriental de la Sierra Madre Occidental en el estado de Zacatecas. Carrasco-Ortiz (2013) registró su presencia a lo largo de la Sierra Madre del Sur en Guerrero y el estado de Oaxaca, y en las sierras de Chiapas. En Nueva Galicia, vive en un rango de elevación que va de 1,240 a 3,200 m y habita en el bosque de coníferas y encinos, bosque mesófilo, bosque tropical caducifolio, matorral xerófilo e incluso en vegetación secundaria como un elemento ruderal. Los registros nuevos en Nueva Galicia representan el extremo noroccidental de su distribución natural (Figura 1).

Ejemplares examinados. México. Jalisco, Ayutla, $1.5 \mathrm{~km}$ en línea recta al SE de Las Iglesias (sobre el camino Santa Mónica-Cacoma), Elev. 2,174 m, 12/08/2012 (fl), P. Carrillo-Reyes y A.S. Monroy 6781 (IBUG). Tapalpa, camino entre Juanacatlán y Tepec, Elev. 2,330 m, 23/08/1986 (fl), A. Rodríguez 584 (IBUG); Rancho Tule, Elev. 2,340 m, 20/09/1998 (fl), M.E. Barba 137 (IBUG). Nayarit, San Pedro Lagunillas, km 11-12 carretera Chapalilla-Compostela, Elev. 1,238 m, 15/08/2013 (fl), A. Castro-Castro y J.G. González-Gallegos 3274 (IBUG, IEB, MEXU, ZEA). Zacatecas, Apozol, $32 \mathrm{~km} \mathrm{~W}$ of Mex. hwy. 41, just S of Jalpa along road to Tlaltenango, Elev. 2,375 m, 08/09/1986 (fl), D.E. Breedlove 63926 (MEXU).

Dahlia wixarika Art.Castro, M.Carrasco-Ortiz \& Aaron Rodr. sp. nov.

D. puganae Aaron Rodr. \& Art. Castro D. dissectae S. Watson et D. scapigerae (A. Dietr.) Knowles \& Westc. affinis sed petiolorum longitudine, caulium et foliorum pubescentia, foliorum magnitudine, foliolorum terminalibus forma, foliolorum alternis praecentia, stipellis praecentia constanter, capitulorum diametro, pedunculorum longitudine, phyllariorum externis numero et forma, florum ligulatis fertilibus praecentia, stigmatibus 2-3-furcatis, et acheniis puberulis tuberculatis et pappo carenti differt.

TIPO: México. Jalisco, municipio de Mezquitic, Cerro del Niño ("Tuurikie"), km 15 del camino Crucero de Santa
Cruz-Santa Clara-San Andrés Cohamiata, Elev. 2,220 m, bosque de pino y encino, 01/10/2011, O. Zuno s.n. (Holotipo: IBUG; Isotipo: MEXU; Figuras 1-4).

Hierba perenne de 0.8-1.0 m alt., escaposa o subescaposa; raíces tuberosas, fasciculadas, fusiformes; tallo fistuloso, estriado, simple, pubescente, sobre todo en los nudos, en ocasiones glabrescentes, 2.0-7.0 mm diám., entrenudos 5-7, (0.5-)2.5-6.0(-17) cm long.; hojas opuestas, ovadas, obovadas o romboides en contorno general, pinnadas o bipinnadas, $7.0-22.5 \times 5.0-19.0 \mathrm{~cm}$, raquis entero; pecíolos sólidos, pulvinulares, conduplicados, enteros, (1.5-)4.0-9.0 $\times 0.2 \mathrm{~cm}$, con pubescencia similar a la del tallo o glabrescentes; estipelas presentes en la base del primer y segundo par de pecíolulos, ocasionalmente también en el tercer par, elípticas, 2.5-20.0 × 1.0-13.0 mm; folíolos 3-7, opuestos, ovados en contorno general, nuevamente pinnados o lobulados, 3.5-9.5 × 1.0-6.0 cm, distantes entre sí (0.7-)3.5$5.5 \mathrm{~cm}$, sésiles en el tercer par de segmentos o con pecíolulos en el primer y segundo par, 0.6-4.6 cm long., el folíolo terminal profundamente trilobado, con el lóbulo central elongado y lanceolado; foliólulos 3-5, alternos o con menos frecuencia opuestos, lanceolados, pero también ovados en contorno general, (0.5-)1.5-3.0 × 0.1-1.2 cm, cuneados o atenuados, acuminados, ciliados, membranáceos, haz de color verde-claro y glabrescente, notablemente más pálidos e híspidos en el envés; capítulos 1-3(-4) por inflorescencia, 2.8-7.0 cm diám. incluidas las lígulas extendidas; pedúnculos 6.0-35.0 cm long., de color púrpura-pardo; involucro campanulado; filarias externas 5-7, erectas o extendidas en antesis, oblanceoladas a romboides, obtusas o agudas, gibosas, 5.0-8.0 × 1.0-3 mm, crasas, de color verde, con 57 nervaduras marcadas de color púrpura; filarias internas 8(-9), lanceoladas, agudas, obtusas, acrescentes, 0.9-1.9 $\times$ 0.2-0.6 cm, de color verde o púrpura en su inserción al receptáculo y translúcidas hacia el ápice, membranáceas, con 7-10 nervaduras marcadas de color púrpura; páleas similares a las filarias internas, pero translúcidas; flores del margen 8, liguladas, fértiles o estériles, tubos glabrescentes, sus láminas ovadas o elípticas, 2.0-3.0 × 0.6-1.7 cm, de color violeta [violeta (16-17) a (4-2), de acuerdo a Kornerup y Wanscher, (1978)], con 6-8 nervaduras paralelas bien marcadas de color violeta, tricomas multicelulares dispersos en la base de las nervaduras, ápices con tres dientes estrechos de 0.5-1.0 mm long.; flores del disco 30-50, tubulosas, 4.7$7.2 \times 0.8-1.2 \mathrm{~mm}$, de color amarillo, tubo $0.9-1.2 \times 0.8 \mathrm{~mm}$, con tricomas dispersos en el ápice, lóbulos de la corola, deltoides, erectos, $0.6 \mathrm{~mm}$ long.; anteras 5, 3.7-4.0 mm long., de color de café, sagitadas, apéndices lanceolados; estilo $2.7 \mathrm{~mm}$ long.; estigma bifurcado en flores del disco, $1.0 \mathrm{~mm}$ long., 2-3-furcado en flores liguladas, en ambas las ramas son fimbriadas, lanceoladas, agudas y abiertas en antesis; aquenios dimórficos, los del margen del receptáculo obovados, 6.0-7.0 × 1.4-1.8 mm, los del centro del receptáculo oblongo-oblanceolados, $6.0-9.0 \times 1.8-2.5 \mathrm{~mm}$, ambos tipos 

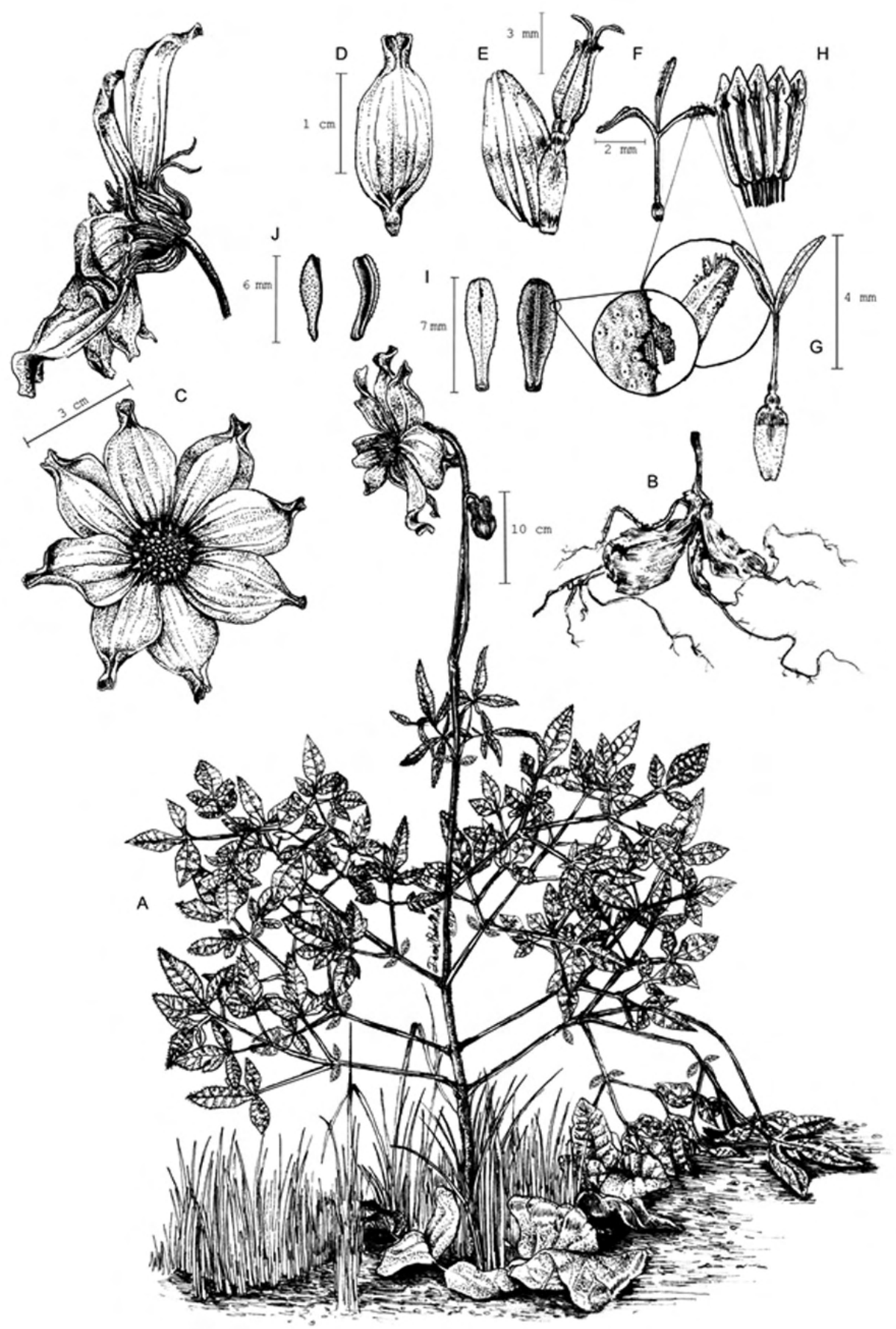

Figura 2. Dahlia wixarika Art. Castro, M. Carrasco-Ortiz \& Aaron Rodr. A) aspecto general, B) raíces; C) vista frontal y lateral de los capítulos, D) flor ligulada, E) flor del disco y pálea, F) estigma trilobado de la flor ligulada, G) estigma de la flor del disco, H) anteras, I) vista frontal y lateral de los aquenios. Ilustración de Daniel Barba López (A-H) y Osvaldo Zuno Delgadillo (I-J).

cóncavos en su cara adaxial, convexos en su cara abaxial, muriculados, grises, glabros o pubérulos, tuberculados; vilano ausente; receptáculo plano, 0.5-0.6 cm diám.

Etimología. El nombre de la especie hace referencia a la etnia Wixárika, también conocida como Huichola.
Distribución y ecología. Dahlia wixarika se conoce de dos poblaciones en el estado de Jalisco y una del estado de Durango, todas restringidas al sistema de montañas conocido como la Sierra de Los Huicholes. Las poblaciones de Jalisco se localizan en el extremo noroeste del municipio de Mezquitic, justo al suroeste de San Andrés Cohamiata, en 
el límite estatal con Nayarit (O. Zuno s.n.; A. Castro-Castro et al. 2983). En una de estas, los individuos son numerosos, se desarrollan a 2,200 m de elevación y en el bosque mixto de Quercus y Pinus. Crecen sobre terrenos con exposición noroeste, escarpados, rocosos y con suelo delgado a casi nulo y pobre en materia orgánica. La segunda población de Jalisco (A. Castro-Castro et al. 2974) crece en un intervalo de elevación entre 1,600 y 1,850 m y se encuentra sobre laderas húmedas de exposición noreste, en el bosque mixto de Quercus y Pinus. Los individuos son saxícolas y el suelo también es delgado o casi nulo, intemperizado y con la roca parental expuesta (Figura 3A-B). En ambas poblaciones, las especies asociadas más notorias son Quercus gentryi C.H.Muller, $Q$. viminea Trel., $Q$. castanea Née, $Q$. eduardii Trel., Pinus lumholtzii B.L.Rob. \& Fernald, P. luzmariae Pérez de la Rosa, P. oocarpa Schiede ex Schltdl., Clethra rosei Britton, Juniperus flaccida Schltdl., Bejaria mexicana Benth., Arctostaphylos pungens Kunth, Iostephane heterophylla (Cav.) Benth., Begonia sp. y Pteridium aquilinum (L.) Kuhn. Otras especies asociadas, Bletia purpurea (Lam.) DC., Dasylirion acrotrichum (Schiede) Zucc., Laelia speciosa (Kunth) Schltr., Mammillaria senilis Lodd. ex SalmDyck y Polianthes platyphylla Rose, están incluidas en la NOM-059-SEMARNAT-2010 (SEMARNAT, 2010), bajo distintas categorías de protección.

En Durango, Dahlia wixarika crece en el centro-sur del estado, en el municipio de Súchil (M. Harker et al., 3995). Esta población es reducida en número de individuos y se desarrolla en suelos rocosos e intemperizados del bosque mixto de Pinus y Quercus. De acuerdo con González-Elizondo et al. (2007) el sitio corresponde a la zona de transición entre las subregiones Pie de Monte-Sierras del Oriente y Sierra Madre Occidental, de la Ecorregión de la Sierra. Las especies asociadas sobresalientes son Arctostaphylos pungens, Comarostaphylis polifolia (Kunth) Zucc. ex Klotzsch, Cosmos concolor Sherff, Dahlia coccinea, Juniperus durangensis Martínez, Muhlenbergia spp., Pinus lumholtzii, Quercus coccolobifolia Trel., Q. radiata Trel., Q. rugosa Née y $Q$. urbanii Trel.

Dahlia wixarika florece entre septiembre y octubre, y fructifica de octubre a noviembre. Su periodo de floración es corto, quizá este comportamiento fenológico evitó su colecta con anterioridad.

Estado de conservación. Con base en los criterios de la Lista Roja de la Unión Internacional para la Conservación de la Naturaleza (IUCN, 2010), Dahlia wixarika está en peligro de extinción [EN, criterio B1c(iv)].

Comentarios taxonómicos. La circunscripción de Dahlia wixarika con las secciones propuestas por Sorensen (1969) es incierta. Por un lado, por desarrollar tallos herbáceos, folíolos opuestos, pecíolos sólidos y filarias externas erectas, D. wixarika puede ser incluida en Dahlia sección Dahlia.
Sin embargo, por sus foliólulos alternos y aquenios tuberculados, se puede relacionar con Dahlia sección Entemophyllon. La combinación de estos caracteres sugiere que la propuesta de Sorensen (1969) no es natural y requiere una revisión. Además, refuerzan los resultados filogenéticos y quimiotaxonómicos obtenidos por Saar et al. (2003a) y Giannasi (1975), respectivamente.

Por su morfología, Dahlia wixarika es afín a $D$. dissecta, D. pugana y D. scapigera (Apéndice 1). Sin embargo, la combinación de los siguientes caracteres permiten reconocerla como un taxon diferente: longitud de los pecíolos, pubescencia de tallos y hojas, dimensiones de las hojas, folíolo terminal oblongo-lanceolado, folíolulos alternos, presencia constante de estipelas, diámetro de los capítulos, longitud de los pedúnculos, número y forma de filarias externas, flores liguladas fértiles, estigmas 2-3-furcados y aquenios dimórficos, pubérulos y tuberculados (Apéndice 1). Se requiere de atención especial en el complejo integrado por D. pugana y D. wixarika. Ambas crecen en simpatría en Mezquitic, Jalisco, y hasta ahora esta es la única zona de contacto conocida entre ellas; sin embargo, sus preferencias ecológicas pueden variar. Dahlia pugana se restringe a las zonas de poca pendiente, suelo profundo y mayor sombra. En contraste, $D$. wixarika crece en laderas rocosas de pendiente pronunciada, con suelo delgado o casi nulo y al sol directo. Además, $D$. wixarika produce hojas mayormente bipinnadas, los folíolulos son alternos, las estipelas están siempre presentes en la base del primer, segundo y tercer par de pecíolulos y la serie externa de filarias se compone por 5-7 piezas (Figura 4). También las características reproductivas son útiles, por ejemplo, en $D$. wixarika las flores liguladas son con mayor frecuencia fértiles, los estigmas son 2-3-furcados y los aquenios son ovados u oblongo-oblanceolados, tuberculados y carecen de vilano (Apéndice 1; Figuras 2-4; ver clave dicotómica).

Ejemplares adicionales examinados. México. Durango, Súchil, San Juan de Michis, km 73 de la brecha hacia Temascal, Elev. 2,779 m, bosque de pino y encino, 08/09/2009 (fl, fr), M. Harker et al. 3995 (CIIDIR, IBUG). Jalisco, Mezquitic, San Andrés Cohamiata, alrededores del poblado, rumbo al desfiladero Los Lirios, Sierra de los Huicholes, Elev. 1,961 m, bosque de pino y encino, 04/09/2012 (fl, fr), A. Castro-Castro et al. 2974 (CIIDIR, IBUG, IEB, MEXU); San Andrés Cohamiata, Cerro del Niño ("Tuurikie"), km 16-18 km, brecha Crucero de Santa Cruz-San Andrés Cohamiata, Elev. 2,223 m, bosque de pino y encino, 04/09/2012 (fl, fr), A. Castro-Castro et al. 2983 (CIIDIR, IBUG, IEB, MEXU).

Novedades en la distribución y macromorfología de Dahlia pugana. De acuerdo con Castro-Castro et al. (2012), D. pugana era conocida solo de su localidad tipo, en un área aproximada de $8 \mathrm{~km}^{2}$ del municipio de Zapopan, Jalisco. En este lugar habita en un rango de elevación de 1,800-1,950 m y crece en cañadas húmedas de exposición noreste sobre suelos 

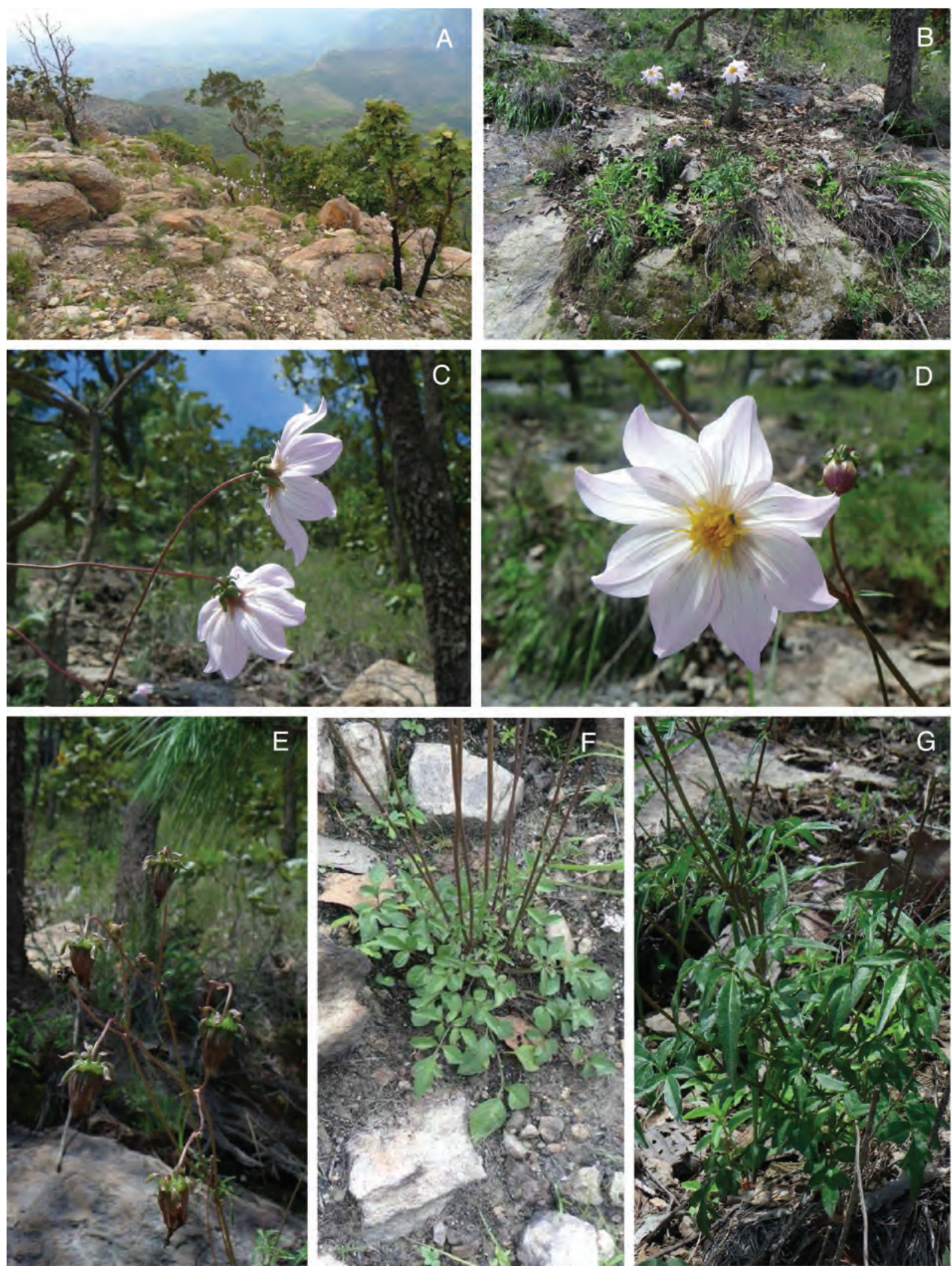

Figura 3. Dahlia wixarika Art. Castro, M. Carrasco-Ortiz \& Aaron Rodr. A) hábitat, B) hábito, C) vista lateral de los capítulos y filarias, D) vista frontal del capítulo, E) capítulos en fructificación, F, G) variabilidad foliar. Fotografías: Osvaldo Zuno-Delgadillo (A, F) y Arturo Castro-Castro (B-E, G). 


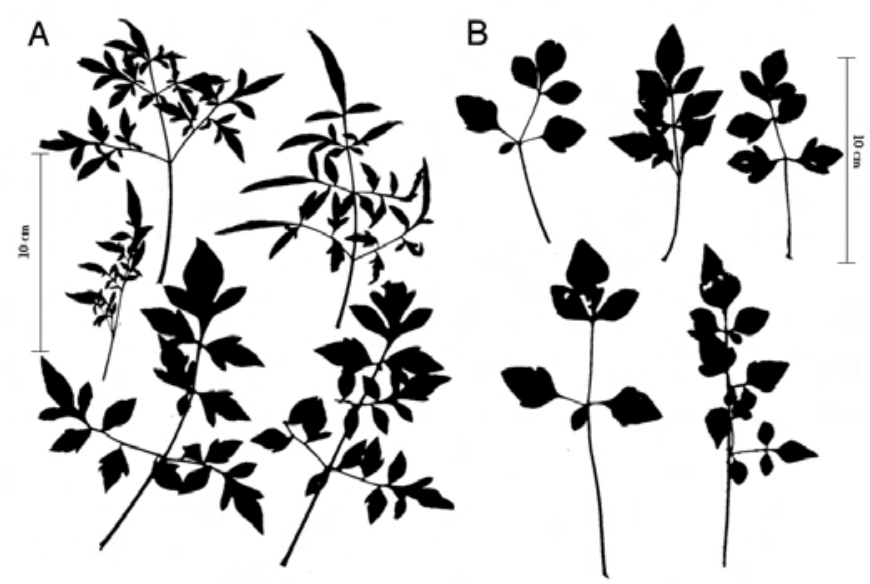

Figura 4. Morfología foliar comparada entre Dahlia wixarika (A) y D. pugana (B).

ácidos del bosque mixto de Quercus y Pinus. La zona constituye la transición entre la Faja Volcánica Transmexicana y la Sierra Madre Occidental. Las exploraciones botánicas y revisiones de herbario recientes, hechas por los autores, han permitido localizar nuevas poblaciones y especímenes.

Las nuevas poblaciones de Dahlia pugana son escasas, pero el número de individuos por población es alto. Una nueva población fue encontrada en el norte de Jalisco, en bosque mixto de Pinus y Quercus de la Sierra de los Huicholes, en la Sierra Madre Occidental y en un rango de elevación de 1,900-2,000 m. También, se encontraron poblaciones y ejemplares antes recolectados en el oeste de Jalisco, en la zona de transición entre la Faja Volcánica Transmexicana y la Sierra Madre del Sur, en la confluencia de las sierras de Cacoma, Jolapa y Verde. En estas últimas el rango de elevación oscila entre 1,800 y 2,110 m (Figura 1).

Morfológicamente, los individuos de las poblaciones del occidente de Jalisco (A. Castro-Castro et al. 2896, 3266, 3272; C.L. Díaz-Luna 5232; J.G. González-Gallegos et al. 1523; M.A. Carrasco-Ortiz et al. 3), varían en la morfología de algunos caracteres en relación con el protólogo de la especie. La talla de las plantas oscila entre 0.5-1.1 m de altura. Las hojas pueden ser pinnadas, bipinnadas, enteras e incluso trifoliadas (C.L. Díaz-Luna 5232), y llegan a medir 9.0-20.0 cm de largo y $4.5-11.0 \mathrm{~cm}$ de ancho, en contorno general. Los folíolos alcanzan $4.5-6.5 \mathrm{~cm}$ de largo. Los pecíolos miden $10.0 \mathrm{~cm}$ de longitud y los pecíolulos $1.5 \mathrm{~cm}$ de longitud. Algunos individuos presentan estipelas en la base del primer y segundo par de pecíolulos. Además, el diámetro de los capítulos alcanza hasta $7.0 \mathrm{~cm}$, incluyendo a las flores liguladas extendidas. Las flores liguladas también difieren en tamaño y color, alcanzan $3.5 \times 1.8 \mathrm{~cm}$ y su color llega a ser de un violeta más intenso o hasta rojizo (A. Castro-Castro et al. 3272).

Ejemplares examinados.- México. Jalisco, Atengo, km 18.5 de la brecha Los Tablones-Soyatlán del Oro, a partir del km 44 de la carretera Ameca-Mascota, Sierra Verde, Elev. 2,110 m, 05/08/2013 (fl, fr), A. Castro-Castro et al. 3272 (IBUG). Atenguillo, La Campana-Volcanes, Elev. 1,800 m, 15/06/1974 (fl), C.L. Díaz-Luna 5232 (GUADA, CIMI). Cuautla, km 31-32 de la carretera Los Volcanes-Cuautla, Tierras Blancas, $1 \mathrm{~km}$ al SO de Fresno Hueco, Elev. 1,860 m, 31/07/2012 (fl), A. Castro-Castro et al. 2896 (IBUG, MEXU, IEB, ZEA); km 38-39 de la carretera San Vicente-Volcanes, entre Tierras Blancas y Fresno Hueco, Elev. 1,931 m, 12/08/2012 (fl, fr), M.A. Carrasco-Ortiz et al. 3 (IBUG, MEXU, IEB, ZEA). El Arenal, parte alta del cerro Los Bailadores, Elev. 1,985 m, 09/09/2012 (fr), M. Leyva-Lozada et al. 164 (IBUG, MEXU). Mezquitic, La Cebolleta, km 16 de la carretera Crucero de Santa Cruz-San Andrés Cohamiata, cerca del Cerro del Niño, Elev. 2,072 m, 04/09/2012 (fl, fr), M.A. Carrasco-Ortiz et al. 7 (IBUG, MEXU, IEB, ZEA). Mixtlán, km 17.5 de la brecha Los Tablones-Soyatlán del Oro, a partir del km 44 de la carretera Ameca-Mascota, Sierra Verde, Elev. 2,072 m, 05/08/2013 (fl, fr), A. Castro-Castro et al. 3266 (IBUG, MEXU, IEB, ZEA); Cerro La Cruz del Crestón, km 14 de la brecha Los Tablones-Soyatlán del Oro, a partir del km 44 de la carretera Ameca-Mascota, Sierra Verde, Elev. 1,912 m, 05/08/2013 (fl), J.G. González-Gallegos et al. 1523 (IBUG).

Clave para la identificación de las especies de Dahlia que crecen en Nueva Galicia, México

1. Filarias externas reflejas o extendidas en antesis.

2. Flores liguladas de color rojo, anaranjado o amarillo .... D. coccinea

2. Flores liguladas de color violeta.

3. Plantas arborescentes o arbustivas, decumbentes, 1.5-

$3 \mathrm{~m}$ alt.; tallos lignificados ............... D. tenuicaulis

3. Plantas herbáceas, erectas, 1.0-1.5 m alt.; tallos no lignificados.

4. Hojas membranáceas; pecíolo, raquis y pecíolulos no alados; filarias externas 9-12 mm long.; flores del disco 50-70 D. sherffii 4. Hojas rígidas y cartáceas; pecíolo, raquis y pecíolulos alados; filarias externas 9-25 mm long.; flores del disco 90-145 D. sorensenii

1. Filarias externas erectas o ascendentes en antesis.

5. Pecíolo y raquis alados y aplanados.

6. Tallos y envés de las hojas vilosos; folíolos anchamente ovados a suborbiculares; capítulos dispuestos en grupos de 2-3 D. barkerae 6. Tallos y hojas glabras o glabriúsculos; folíolos romboides a lanceolados; capítulos solitarios D. scapigera

5. Pecíolo y raquis no alados y conduplicados. 7. Hojas pinnadas; folíolulos opuestos; flores liguladas estériles; estigmas de las flores liguladas 2furcados; filarias externas 5; aquenios lisos; vilano discoide o ausente ........................... p. pugana 
7. Hojas bipinnadas; folíolulos alternos; flores liguladas fértiles; estigmas de las flores liguladas 2-3furcados; filarias externas 5-7; aquenios tuberculados; vilano ausente D. wixarika

\section{Agradecimientos}

Este trabajo fue financiado por el Consejo Nacional de Ciencia y Tecnología (CONACyT) mediante el proyecto 80200, convocatoria CB-2007-01. Agradecemos el equipo otorgado por la organización Idea Wild. Agradecemos las valiosas aportaciones de dos revisores anónimos durante el proceso de arbitraje. Se agradece a Jesús G. González Gallegos su ayuda en la elaboración de la diagnosis en latín. Gracias a Guadalupe Munguía Lino, Lesslie Martínez Rubí, Alfredo Frías Castro, Esteban A. Suárez Muro, Irving Rosas Ruiz y Jesús G. González Gallegos por su apoyo y ayuda durante el trabajo de campo. Asimismo, se agradece al personal de los herbarios CIIDIR, CIMI, ENCB, GUADA, IBUG, IEB, MEXU y ZEA, por las facilidades concedidas durante la consulta de sus colecciones. Karina Castro Castro y Daniel Barba López merecen nuestro agradecimiento por su apoyo en la edición de imágenes e ilustración de Dahlia wixarika, respectivamente. Gracias a Judith Morales Saavedra por la ayuda en la adquisición de literatura. Por último, se agradece a la comunidad Wixárika de San Andrés Cohamiata, Mezquitic, Jalisco, en especial a Rosalio Sánchez "Chalio" y a Don Santos, del pueblo de San Juan de Míchis, Súchil, Durango, por la hospitalidad concedida durante el trabajo de campo.

\section{Literatura citada}

Carrasco-Ortiz M.A. 2013. Distribución geográfica y riqueza del género Dahlia Cav. (Asteraceaea) en México. Tesis de licenciatura, División de Ciencias Biológicas y Ambientales, Centro Universitario de Ciencias Biológicas y Agropecuarias, Universidad de Guadalajara, Guadalajara. 76 pp.

Castro-Castro A., Rodríguez A., Vargas-Amado G. y Harker M. 2012. Diversidad del género Dahlia (Asteraceae: Coreopsideae) en Jalisco, México y descripción de una especie nueva. Revista Mexicana de Biodiversidad 83:347-358.

ESRI. 2002. ArcView 3.3. Environmental Systems Research Institute, Inc., California.

Gatt M.K., Hammett K.R.W. y Murray B.G. 2000. Molecular phylogeny of the genus Dahlia Cav. (Asteraceae, HeliantheaeCoreopsidinae) using sequences derived from the internal transcribed spacers of nuclear ribosomal DNA. Botanical Journal of the Linnean Society 133:229-239.

Gatt M., Ding H., Hammett K. y Murray B. 1998. Polyploidy and evolution in wild and cultivated Dahlia species. Annals of Botany 81:647-656.

Giannasi D.E. 1975. Flavonoid chemistry and evolution in Dahlia (Compositae). Bulletin of the Torrey Botanical Club 102:404412.

González-Elizondo M.S., González-Elizondo M. y Márquez-Lina- res M.A. 2007. Vegetación y Ecorregiones de Durango. Plaza y Valdés Editores, Centro Interdisciplinario de Investigación para el Desarrollo Integral Regional, Instituto Politécnico Nacional, México, D.F.

Hansen H.V. y Hjerting J.P. 1996. Observations on chromosome numbers and biosystematics in Dahlia (Asteraceae, Heliantheae) with an account on the identity of D. pinnata, D. rosea, and D. coccinea. Nordic Journal of Botany 16:445-455.

Hansen H.V. y Hjerting J.P. 2000. The Early History of the Domestication of Dahlia (Asteraceae, Heliantheae) with Emphasis on the Period 1791-1836: Including Observations on Taxonomy, Chromosome Numbers, Biochemistry, Biosystematics, Ray Colour Inheritance, and Ray Colour Designation within the Genus. The Botanical Garden University of Copenhagen, Copenague.

Hansen H.V. y Sørensen P.D. 2003. A new species of Dahlia (Asteraceae, Coreopsideae) from Hidalgo State, Mexico. Rhodora 105:101-105.

IUCN. International Union for Conservation of Nature. 2012. Categorías y criterios de la Lista Roja de la IUCN. Versión 3.1. $2^{\mathrm{a}}$ ed. International Union for Conservation of Nature. Cambridge.

Kornerup A. y Wanscher J.H. 1978. Methuen Handbook of Colour. $3^{\text {a }}$ ed. Methuen, Londres.

McVaugh R. 1961. Euphorbiaceae novae Novo-Galicianae. Brittonia 13:145-205.

McVaugh R. 1972. Botanical exploration in Nueva Galicia, Mexico; from 1790 to the present time. Contributions of the University of Michigan Herbarium 9:205-357.

McVaugh R. 1984. Compositae. Flora Novo-Galiciana 12:1-1157.

Saar D.E. 2002. Dahlia neglecta (Asteraceae, Coreopsideae), a new species from Sierra Madre Oriental, Mexico. Sida 20:593-596.

Saar D.E. y Sørensen P.D. 2000. Dahlia parvibracteata (Asteraceae, Coreopsideae), a new species from Guerrero, Mexico. Novon 10:407-410.

Saar D.E. y Sørensen P.D. 2005. Dahlia sublignosa (Asteraceae): a new species in its own right. Sida 21:2161-2167.

Saar D.E., Sørensen P.D. y Hjerting J.P. 2002. Dahlia spectabilis (Asteraceae, Coreopsideae), a new species from San Luis Potosí, Mexico. Brittonia 54:116-119.

Saar D.E., Polans N.O. y Sørensen P.D. 2003a. A phylogenetic analysis of the genus Dahlia (Asteraceae) based on internal and external transcribed spacer regions of nuclear ribosomal DNA. Systematic Botany 28:627-639.

Saar D.E., Sørensen P.D. y Hjerting J.P. 2003b. Dahlia campanulata and D. cuspidata (Asteraceae, Coreopsideae): two new species from México. Acta Botanica Mexicana 64:19-24.

SEMARNAT. Secretaría de Medio Ambiente y Recursos Naturales. 2010. Norma Oficial Mexicana NOM-059-ECOL-2010, Protección ambiental-Especies nativas de México de flora y fauna silvestres-Categorías de riesgo y especificaciones para su inclusión, exclusión o cambio-Lista de especies en riesgo.

Sorensen P.D. 1969. Revision of the genus Dahlia (Compositae, Heliantheae-Coreopsidinae). Rhodora 71:309-365, 367-416.

Sorensen P.D. 1970. The Dahlia: an early history. Arnoldia 30:121138.

Sørensen P.D. 1980. New taxa in the genus Dahlia (Asteraceae, Heliantheae-Coreopsidinae). Rhodora 82:353-360.

Sørensen P.D. 1987. Dahlia congestifolia, section Entemophyllon (Asteraceae: Heliantheae, Coreopsidinae), new from Hidalgo, Mexico. Rhodora 89:197-203. 
Thiers B. 2013. Index Herbariorum. New York Botanical Garden's Virtual Herbarium. <http://sweetgum.nybg.org/ih/> (consultado 8 abril 2013).

Treviño de Castro G., Mera O.L.M., Bye B.R., Mejía M.J.M. y Laguna C.A. 2007. Historia de la Dalia (Acocoxóchitl), la flor nacional de México. Secretaría de Agricultura, Ganadería, Desarrollo Rural, Pesca y Alimentación, Servicio Nacional de Inspección y Certificación de Semillas, Sistema Nacional de Recursos Fitogenéticos para la Alimentación y la Agricultura, Red de Ornamentales (Grupo Dahlia), Estado de México.

Recibido: 18 de diciembre de 2013

Aceptado: 13 de abril de 2014 
Apéndice 1. Morfología comparada entre Dahlia wixarika y especies afines. Hábitat: $\mathrm{BPE}=$ bosque mixto de pino y encino; $\mathrm{BEP}=$ bosque mixto de encino y pino; $\mathrm{BC}=$ bosque de coníferas; $\mathrm{MX}=$ matorral xerófilo.

\begin{tabular}{|c|c|c|c|c|}
\hline Carácter & D. wixarika & D. dissecta & D. pugana & D. scapigera \\
\hline Hábito & $\begin{array}{l}\text { hierba erecta, } 0.8-1 \mathrm{~m} \text {, } \\
\text { sin ramificarse, escaposa } \\
\text { o subescaposa }\end{array}$ & $\begin{array}{l}\text { hierba erecta o subarbustiva, } \\
\text { 1.0-1.2 m, con múltiples } \\
\text { ramificaciones }\end{array}$ & $\begin{array}{l}\text { hierba erecta o postrada, } \\
0.4-0.7 \mathrm{~m} \text {, sin ramificarse, } \\
\text { escaposa o subescaposa }\end{array}$ & $\begin{array}{l}\text { hierba erecta, 0.6-0.9 } \\
(-1.8) \mathrm{m} \text {, sin ramificarse, } \\
\text { escaposa o subescaposa }\end{array}$ \\
\hline Entrenudos & $(0.5-) 2.5-6.0(-17) \mathrm{cm}$ & $0.5-3.8 \mathrm{~cm}$ & $2.0-4.0(-5.0) \mathrm{cm}$ & $0.3-3.0(-10.0) \mathrm{cm}$ \\
\hline Tallos & $\begin{array}{l}\text { pubescentes, sobre todo } \\
\text { en los nudos, en ocasiones } \\
\text { glabrescentes }\end{array}$ & glabros & pubescentes a velutinos & glabros \\
\hline Hojas & $\begin{array}{l}\text { pinnadas o bipinnadas, } \\
7.0-22.5 \times 5.0-19.0 \mathrm{~cm}\end{array}$ & $\begin{array}{l}\text { bipinnadas o tripinnadas, } \\
15.0-28.0 \times 14.0-27.0 \mathrm{~cm}\end{array}$ & $\begin{array}{l}\text { pinnadas, bipinnadas, } \\
\text { simples o trifoliadas, } \\
(4.5-) 9.0-14.0(-20.0) \times \\
4.0-11.0 \mathrm{~cm}\end{array}$ & $\begin{array}{l}\text { pinnadas, trifoliadas } \\
\text { o enteras, } 15.0-30.0 \times \\
6.0-11.0 \mathrm{~cm}\end{array}$ \\
\hline Pecíolo & $\begin{array}{l}\text { conduplicado, entero, } \\
(1.5-) 4.0-9.0 \mathrm{~cm}\end{array}$ & $\begin{array}{l}\text { conduplicado, entero, } \\
4.0-13.0 \mathrm{~cm}\end{array}$ & $\begin{array}{l}\text { conduplicado, entero, } \\
(2.5-) 3.5-5.5(-10.0) \mathrm{cm}\end{array}$ & $\begin{array}{l}\text { aplanado, alado, } \\
6.0-15.0 \mathrm{~cm}\end{array}$ \\
\hline $\begin{array}{l}\text { Número e } \\
\text { indumento } \\
\text { de folíolos }\end{array}$ & $\begin{array}{l}\text { 3-7, haz glabrescente, } \\
\text { envés hispido }\end{array}$ & $\begin{array}{l}\text { 5-9, glabros a glabrescentes } \\
\text { en ambas caras }\end{array}$ & $\begin{array}{l}3-5(-7), \text { pubescentes a } \\
\text { velutinos en ambas caras }\end{array}$ & $\begin{array}{l}(3-) 5-7(-9), \text { glabros a } \\
\text { glabrescentes en ambas } \\
\text { caras }\end{array}$ \\
\hline $\begin{array}{l}\text { Folíolo } \\
\text { terminal }\end{array}$ & $\begin{array}{l}\text { disimilar a los laterales, } \\
\text { profundamente trilobado, } \\
\text { con el lóbulo central } \\
\text { elongado y lanceolado }\end{array}$ & similar a los laterales & $\begin{array}{l}\text { similar o de tamaño } \\
\text { ligeramente mayor que } \\
\text { los laterales }\end{array}$ & $\begin{array}{l}\text { más largo que } \\
\text { los laterales }\end{array}$ \\
\hline Folíolulos & alternos, a veces opuestos & alternos & opuestos & ausentes \\
\hline Estipelas & $\begin{array}{l}\text { presentes en la base del } \\
\text { primer, segundo y tercer } \\
\text { par de pecíolulos }\end{array}$ & $\begin{array}{l}\text { ausentes o presentes en } \\
\text { la base del primer par } \\
\text { de pecíolulos }\end{array}$ & $\begin{array}{l}\text { ausentes, pero en } \\
\text { ocasiones presentes en la } \\
\text { base del primer y segundo } \\
\text { par de pecíolulos }\end{array}$ & ausentes \\
\hline $\begin{array}{l}\text { Número de } \\
\text { capítulos }\end{array}$ & $\begin{array}{l}\text { 1-3(-4), solitarios o } \\
\text { varios contemporáneos }\end{array}$ & $1-3$, uno a la vez & $\begin{array}{l}(1-) 3, \text { por lo general } \\
\text { uno a la vez }\end{array}$ & $\begin{array}{l}\text { 1-3(-6), solitarios o } \\
\text { varios contemporáneos }\end{array}$ \\
\hline $\begin{array}{l}\text { Diámetro de } \\
\text { capítulos, } \\
\text { incluidas las } \\
\text { lígulas } \\
\text { extendidas }\end{array}$ & $2.8-7.0 \mathrm{~cm}$ & $4.7-5.0 \mathrm{~cm}$ & $5.5-7.0 \mathrm{~cm}$ & $5.0-7.5 \mathrm{~cm}$ \\
\hline Pedúnculos & $6.0-35.0 \mathrm{~cm}$ & $10.0-35.0 \mathrm{~cm}$ & $20.0-50.0 \mathrm{~cm}$ & $22.0-55.0 \mathrm{~cm}$ \\
\hline $\begin{array}{l}\text { Filarias } \\
\text { externas }\end{array}$ & $\begin{array}{l}5-7,5.0-8.0 \times 1.0-3 \mathrm{~mm} \text {, } \\
\text { oblanceoladas a } \\
\text { romboides, } 5-7 \text { nervadas, } \\
\text { erectas o extendidas }\end{array}$ & $\begin{array}{l}5,5.0-7.0 \times 2.0-4.0 \mathrm{~mm} \text {, } \\
\text { oblongo a obovadas o } \\
\text { suborbiculares, } 3-5 \text { nervadas, } \\
\text { erectas o algo extendidas }\end{array}$ & $\begin{array}{l}5,5.0-7.0 \times 1.5-2.5 \mathrm{~mm} \text {, } \\
\text { lanceoladas, } 3-5 \text { nervadas, } \\
\text { erectas }\end{array}$ & $\begin{array}{l}5,5.0-11.0 \times 1.5-3.0 \mathrm{~mm} \text {, } \\
\text { ensiformes, } 1-5 \text { nervadas, } \\
\text { erectas o extendidas }\end{array}$ \\
\hline
\end{tabular}


Novedades en Dahlia Para la Nueva Galicia, MéXico

Apéndice 1. Continuación

\begin{tabular}{|c|c|c|c|c|}
\hline Carácter & D. wixarika & D. dissecta & D. pugana & D. scapigera \\
\hline $\begin{array}{l}\text { Filarias } \\
\text { internas }\end{array}$ & $\begin{array}{l}\text { 8(-9), 9.0-19.0 × } \\
\text { 2.0-6.0 mm, de color } \\
\text { verde-púrpura en su } \\
\text { inserción al receptáculo y } \\
\text { translúcidas hacia el ápice }\end{array}$ & $\begin{array}{l}8,9.0-20.0 \times 2.0-5.0 \mathrm{~mm} \\
\text { de color café, hialinas }\end{array}$ & $\begin{array}{l}8(-10), 12.0-15.0 \times \\
4.0-4.5 \mathrm{~mm} \text {, de color } \\
\text { verde-translúcido }\end{array}$ & $\begin{array}{l}7-8,0.6-16.0 \times \\
3.0-7.5 \mathrm{~mm} \text {, de color } \\
\text { café, hialinas }\end{array}$ \\
\hline $\begin{array}{l}\text { Flores del } \\
\text { margen }\end{array}$ & $\begin{array}{l}\text { fértiles, algunas estériles, } \\
6-8 \text { nervadas, de color } \\
\text { violeta }\end{array}$ & $\begin{array}{l}\text { estériles, 5-7 nervadas, de } \\
\text { color violeta o blanco }\end{array}$ & $\begin{array}{l}\text { estériles, 2-4 nervadas, } \\
\text { de color violeta, violeta- } \\
\text { intenso o rojizo }\end{array}$ & $\begin{array}{l}\text { fértiles, 3-5 nervadas, } \\
\text { de color violeta o } \\
\text { blanco }\end{array}$ \\
\hline $\begin{array}{l}\text { Estigma de } \\
\text { flores del } \\
\text { margen }\end{array}$ & 2-3-furcado & bifurcado & bifurcado & bifurcado \\
\hline $\begin{array}{l}\text { Flores del } \\
\text { disco }\end{array}$ & $25-50$ & $35-50$ & $20-55$ & $35-60$ \\
\hline Aquenios & $\begin{array}{l}\text { dimórficos, ovados a } \\
\text { oblongo-oblanceolados, } \\
6.0-9.0 \times 1.4-2.5 \mathrm{~mm} \text {, de } \\
\text { color gris, muriculados, } \\
\text { pubérulos, tuberculados }\end{array}$ & $\begin{array}{l}\text { homomórficos, linear- } \\
\text { oblanceolados, } 8.0-11.0 \times \\
1.0-2.8 \mathrm{~mm} \text {, de color } \\
\text { negruzco, hispídulos, } \\
\text { muriculados, finamente } \\
\text { tuberculados }\end{array}$ & $\begin{array}{l}\text { dimórficos, ovados o } \\
\text { elípticos, } 4.0-8.0 \times \\
\text { 2.0-2.5 mm, de color gris, } \\
\text { pubérulos, muriculados }\end{array}$ & $\begin{array}{l}\text { homomórficos, linear- } \\
\text { oblanceolados, } 5.0-8.0 \times \\
3.0-3.5 \mathrm{~mm} \text {, de color } \\
\text { negro, pubérulos, } \\
\text { muriculados }\end{array}$ \\
\hline Vilano & ausente & $\begin{array}{l}\text { ausente o en forma de dos } \\
\text { proyecciones diminutas }\end{array}$ & ausente o discoide & $\begin{array}{l}\text { ausente o en forma de dos } \\
\text { proyecciones diminutas }\end{array}$ \\
\hline Floración & septiembre-octubre & julio-octubre & julio-agosto & julio-octubre \\
\hline $\begin{array}{l}\text { Rango de } \\
\text { elevación (m) }\end{array}$ & $1,960-2,780$ & $2,100-2,260$ & $1,800-2,110$ & $2,000-3,350$ \\
\hline Hábitat & BPE, BEP & $M X, B C$ & BPE, BEP & BPE \\
\hline $\begin{array}{l}\text { Distribución } \\
\text { geográfica }\end{array}$ & Durango, Jalisco & Hidalgo, Querétaro & Jalisco & $\begin{array}{l}\text { Distrito Federal, } \\
\text { Guanajuato, Hidalgo, } \\
\text { Estado de México, } \\
\text { Michoacán }\end{array}$ \\
\hline
\end{tabular}

